\title{
Le troisième récepteur de I'histamine : pharmacologie et perspectives thérapeutiques
}

Le troisième récepteur de l'histamine a d'abord été découvert et étudié dans le cerveau où il contrôle la synthèse et la libération de l'amine elle-même. Il pourrait aussi agir sur la libération de sérotonine. Un puissant agoniste et un puissant antagoniste de ce récepteur ont été synthétisés ; le premier a une action sédative alors que le second stimule la vigilance. Ces récepteurs ont également été détectés dans le poumon et le tractus digestif où ils jouent un rôle inhibiteur sur les influx cholinergiques. De ce fait, les agonistes spécifiques pourraient aussi avoir des indications thérapeutiques en pneumologie (traitement du bronchospasme et de la réaction inflammatoire de l'asthme) et, peut-être, en gastro-entérologie (action antispasmodique et antisécrétoire).

\section{Philippe Devillier Jean-Michel Arrang Monique Garbarg Hélène Pollard Jean-Charles Schwartz}

$\mathrm{Ph}$. Devillier : docteur en médecine. Laboratoire Bioprojet, 30, rue des Francs-Bourgeois, 75003 Paris, France.

J.-M. Arrang : docteur ès sciences. M. Garbarg docteur ès sciences. H. Pollard: docteur ès sciences. J.-C. Schwartz: docteur ès sciences. Inserm U. 109, neurobiologie et pharmacologie, centre Paul-Broca, 2 ter, rue d'Alésia, 75014 Paris, France.

\section{TIRÉS A PART}

'histamine fait partie de ces messagers ubiquitaires intervenant dans la régulation de processus physiologiques très variés dans les domaines immuno-allergologiques, cardiovasculaires, digestifs et, enfin, comme neuromédiateur dans le cerveau [1]. Pendant longtemps, on a considéré que ses puissantes actions sur les diverses cellules cibles se développaient exclusivement par l'intermédiaire de deux récepteurs dénommés $\mathrm{H}_{1}$ et $\mathrm{H}_{2}$. Il est inutile de souligner comment la mise au point d'antagonistes $\mathrm{H}_{1}$ et $\mathrm{H}_{2}$ a déjà fourni à la thérapeutique des médicaments majeurs, ainsi que nous l'a encore rappelé en 1988 l'attribution du prix Nobel de médecine à sir J. Black, découvreur du récepteur $\mathrm{H}_{2}$. Récemment, la découverte d'un troisième récepteur de l'histamine [2], suivie de la mise au point d'agents susceptibles soit de le stimuler soit de le bloquer puissamment et sélectivement [3] a, tout naturellement, ouvert des perspectives théra- peutiques nouvelles. Initialement identifié dans le cerveau comme un autorécepteur, c'est-à-dire un récepteur régulant la synthèse et la libération de l'amine par elle-même, le récepteur $\mathrm{H}_{3}$ s'est récemment révélé avoir des rôles beaucoup plus larges puisqu'il a été aussi localisé sur des neurones non histaminergiques du cerveau et même dans divers organes périphériques. Ce sont ces progrès récents et les applications thérapeutiques qui devraient en découler que nous nous proposons d'analyser ici.

\section{Le récepteur $\mathrm{H}_{3}$ : nouveaux ligands pour une nouvelle cible pharmacologique}

C'est sur un modèle simple de libération de l'histamine neuronale par des coupes de cerveau de rat qu'a été initialement observé le phénomène d'auto-inhibition: l'amine radioactive, synthétisée à partir de son précurseur ${ }^{3} \mathrm{H}$, est libérée en moindre quantité par un stimulus dépolari- 
sant lorsqu'on ajoute au milieu de l'histamine non radioactive. L'autoinhibition est prononcée puisqu'elle est voisine de $60 \%$ si la libération est induite par le stimulus $\mathrm{K}^{+}$[2] et peut même être totale si une dépolarisation électrique de courte durée est imposée[4]. Ce frein puissant qu'exerce l'amine sur sa propre libération met bien en jeu un récepteur comme l'indiquent les effets semblables de quelques analogues de l'histamine et la réversion compétitive exercée par un certain nombre de composés. Or les antihistaminiques $\mathrm{H}_{1}$ sont totalement inefficaces sur ce modèle et, si des antihistaminiques $\mathrm{H}_{2}$ présentent un certain pouvoir antagoniste, celui-ci (mesuré par les constantes de dissociation apparentes) est clairement différent de celui qu'ils exercent sur les récepteurs $\mathrm{H}_{2}$ (Tableau I). Cette «carte d'identité pharmacologique » du récepteur diffère donc de celles, bien établies, des récepteurs $\mathrm{H}_{1}$ et $\mathrm{H}_{2}$ et nous a naturellement conduit à proposer la dénomination « $\mathrm{H}_{3}$ » pour l'autorécepteur [2]. Cette dénomination nouvelle est maintenant largement adoptée car, comme il se doit, elle est fondée sur une différence non de localisation ou de fonction mais de pharmacologie (encore que la génétique moléculaire soit en train de constituer une discipline plus prolifique que la pharmacologie pour mettre au monde de nouveaux récepteurs !).

Mais l'existence du récepteur $\mathrm{H}_{3}$ n'a pu être établie de manière définitive qu'avec la mise au point d'agents hautement spécifiques de l'autorécepteur; n'interagissant que de manière marginale avec les récepteurs $\mathrm{H}_{1}$ et $\mathrm{H}_{2}$ [3]. Dans cette mise au point progressive, effectuée en collaboration avec deux laboratoires universitaires de pharmacochimie, celui de l'université libre de Berlin en RFA (Pr W. Schunack) et celui de l'université de Caen (Pr M. Robba), le point de départ a été la molécule d'histamine elle-même.

Ce choix s'imposait, car une des caractéristiques pharmacologiques les plus frappantes du récepteur $\mathrm{H}_{3}$ réside dans sa grande « sensibilité » à l'histamine. En effet, son activation requiert des concentrations de l'amine environ cent fois plus faibles que dans le cas des récepteurs $\mathrm{H}_{1}$ et $\mathrm{H}_{2}$, $\mathrm{m} / \mathrm{s} n^{\circ} 6$ vol. 5 , juin 89

\begin{tabular}{|c|c|c|c|}
\hline \multicolumn{4}{|c|}{$\begin{array}{l}\text { Tableau I } \\
\text { EFFETS DE DIFFÉRENTS COMPOSÉS HISTAMINIQUES SUR } \\
\text { LES TROIS CLASSES DE RÉCEPTEURS DE L'HISTAMINE }\end{array}$} \\
\hline & Récepteur $\mathrm{H}_{3}$ & Récepteur $\mathrm{H}_{1}$ & Récepteur $\mathbf{H}_{2}$ \\
\hline Agonistes & \multicolumn{3}{|c|}{ Puissance relative (\%) } \\
\hline $\begin{array}{l}\text { Histamine } \\
\text { (R) } \alpha \text {-méthylhistamine } \\
\text { (S) } \alpha \text {-méthylhistamine }\end{array}$ & $\begin{array}{r}100 \\
1550 \\
13\end{array}$ & $\begin{array}{r}100 \\
0,5 \\
0,5\end{array}$ & $\begin{array}{c}100 \\
1 \\
1,7\end{array}$ \\
\hline Antagonistes & \multicolumn{3}{|c|}{ Constante de dissociation, $\mathrm{Ki}$ (nM) } \\
\hline $\begin{array}{l}\text { Mépyramine } \\
\text { Burimamide } \\
\text { Cimétidine } \\
\text { Thiopéramide }\end{array}$ & $\begin{array}{l}>3000 \\
70 \\
33000 \\
4,3\end{array}$ & $\begin{array}{c}0,4 \\
290000 \\
450000 \\
>100000\end{array}$ & $\begin{aligned} & 7,800 \\
& 790 \\
&> 10000\end{aligned}$ \\
\hline
\end{tabular}

suggérant que la molécule d'histamine est plus étroitement reconnue par le récepteur $\mathrm{H}_{3}$. De fait, l'établissement de relations structure-activité a rapidement montré que la stéréosélectivité (c'est-à-dire la faculté de différencier des isomères optiques) [5] et, d'une manière plus générale, la sélectivité du récepteur $\mathrm{H}_{3}$ vis-à-vis des agonistes est beaucoup plus grande que celle des deux autres récepteurs. Seule est tolérée l'introduction de modifications très discrètes dans la molécule d'histamine pour que le récepteur $\mathrm{H}_{3}$ 《 confonde » ces molécules modifiées. Notre travail de sculpture progressive de la molécule d'agoniste nous a finalement conduit à un composé, la $(\mathrm{R}) \alpha$ méthylhistamine (Tableau I), présentant des caractéristiques particulièrement intéressantes : très forte activité (le composé agissant à des concentrations nanomolaires est quinze fois plus actif que l'histamine), très forte stéréosélectivité (l'inverse optique est cent fois moins actif que l'isomère $R$ ) et, surtout, très forte spécificité pharmacologique.

Cette dernière caractéristique, très importante pour les utilisations expérimentales et cliniques de la molécule, doit être soulignée puisque la (R) $\alpha$-méthylhistamine présente une activité négligeable vis-àvis des récepteurs $\mathrm{H}_{1}$ et $\mathrm{H}_{2}$, et nulle vis-à-vis des divers récepteurs monoaminergiques.

C'est par une démarche analogue que nous avons mis au point une série d'antagonistes $\mathrm{H}_{3}$ originaux et très puissants, dont le prototype est le thiopéramide, actif à des concentrations nanomolaires et présentant, lui aussi, une très haute spécificité pharmacologique (Tableau I). A ce propos, il est intéressant de souligner qu'un certain nombre d'antagonistes $\mathrm{H}_{2}$, en particulier les «ancêtres » de la cimétidine (burimamide et métiamide) possèdent une affinité non négligeable vis-à-vis des récepteurs $\mathrm{H}_{3}$ : ainsi certaines activités biologiques de l'histamine, qui dans le passé avaient été (trop hâtivement) attribuées au récepteur $\mathrm{H}_{2}$, sont vraisemblablement à mettre au compte du récepteur $\mathrm{H}_{3}$.

Tout nouvel agent pharmacologique sélectif, avant même de devenir un médicament, constitue un agent d'investigation précieux et l'utilisation dans divers laboratoires de la $(R)$ $\alpha$-méthylhistamine et du thiopéramide a déjà permis d'analyser plusieurs mécanismes biologiques et de prévoir des applications thérapeutiques.

\section{Des récepteurs $\mathrm{H}_{3}$ dans le cerveau}

Le cerveau comporte un millier de neurones à histamine présents dans le noyau tubéromammillaire de l'hypothalamus postérieur d'où émanent des projections diffuses dans l'ensemble de l'encéphale [1]. Cette disposition particulière, rappelant étrangement celles des autres sys- 


\section{RÉFÉRENCES}

1. Pollard H, Arrang JM, Garbarg M, Schwartz JC. Les transmissions histaminergiques cérébrales. médecine/sciences 1986; 1 . $10-6$.

2. Arrang JM, Garbarg M, Schwartz JC. Autoinhibition of brain histamine release mediated by a novel class $\left(\mathrm{H}_{3}\right)$ of histamine receptor. Nature $1983 ; 302$ : 832-7.

3. Arrang JM, Garbarg M, Lancelot JM, et al. Highly potent and selective ligands for histamine $\mathrm{H}_{3}$-receptors. Nature 1987; 327 : $117-23$

4. Van der Werf JF, Bast A, Bijloo GJ, Van der Vliet A, Timmerman H. HA autoreceptor assay with superfused slices of rat brain cortex and electrical stimulation. Eur I Pharmacol 1987; 138 : 199-206.

5. Arrang JM, Schwartz JC, Schunack W. Stereoselectivity of the histamine $\mathrm{H}_{3}$-presynaptic autoreceptor. Eur J Pharmacol 1985 ; $117: 109-14$

6. Arrang JM, Garbarg M, Schwartz JC. Autoregulation of histamine release in brain by presynaptic $\mathrm{H}_{3}$-receptors. Neuroscience $1985 ; 15: 553-62$.

7. Arrang JM, Garbarg M, Schwartz JC. Autoinhibition of histamine synthesis mediated by presynaptic $\mathrm{H}_{3}$-receptors. Neuroscience $1987 ; 23$ : 149-57.

8. Garbarg M, Trung Tuong MD, Gros C, Schwartz JC. Effects of histamine $\mathrm{H}_{3}$-receptor ligands on various biochemical indices of histaminergic neuron activity in rat brain. Eur J Pharmacol 1989 (sous presse).

9. Jouvet M, Sakai K, Lin JS. Role of histaminergic neurons in the regulation of arousal in the cat. Eur J Neurosci 1988; 5 (suppl.): 5 .

10. Bristow LJ, Bennett GW. A role for histamine $\mathrm{H}_{3}$-receptors in histamine-induced hypoactivity in the rat. Br J Pharmacol 1988; 94 : 319.

11. Arrang JM, Devaux B, Chodkiewicz JP, Schwartz JC. $\mathrm{H}_{3}$-receptors control histamine release in human brain. $J$ Neurochem 1988 ; 51 : 105-8.

12. Arrang JM, Garbarg M, Quach TT, Trung Tuong MD, Yeramian E, Schwartz JC. Actions of betahistine at histamine receptors in the brain. Eur J Pharmacol 1985; 111 : 73 84.

13. Schlicker E, Betz R, Göther M. Histamine $\mathrm{H}_{3}$ receptor-mediated inhibition of serotonin release in the rat brain cortex. NaunynSchmiedeb. Arch Pharmacol 1988 ; 337 : 588- tèmes mono-aminergiques (catécholaminergiques et sérotoninergiques) est, comme dans le cas de ces derniers, particulièrement adaptée à un contrôle coordonné de l'excitabilité de vastes aires cérébrales. De fait, de nombreux arguments expérimentaux indiquent que les fonctions des neurones histaminergiques présentent des analogies avec celles des autres systèmes monoaminergiques : contrôle des états de vigilance, du débit sanguin et du métabolisme énergétique cérébral, régulation de la sécrétion des hormones hypophysaires, etc. Cependant, jusqu'ici, on manquait largement d'outils pharmacologiques pour moduler expérimentalement l'activité des systèmes histaminergiques cérébraux et, ainsi, établir leurs fonctions. L'agoniste et l'antagoniste $\mathrm{H}_{3}$ sont en train de combler ce vide.

A l'aide de ceux-ci, on a pu vérifier que les récepteurs $\mathrm{H}_{3}$ sont bien localisés sur les terminaisons (et probablement aussi les corps cellulaires) des neurones histaminergiques du cerveau où ces récepteurs présynaptiques exercent un frein tonique sur la libération mais aussi la biosynthèse du neurotransmetteur [6, 7]. Ces caractéristiques, d'abord mises en évidence in vitro sur des terminaisons nerveuses isolées, ont pu être vérifiées chez l'animal vivant car, bien absorbés par voie orale, aussi bien l'agoniste que l'antagoniste $\mathrm{H}_{3}$ franchissent la barrière hématoencéphalique. De fait, à faible dose et de manière prolongée, ces deux composés modifient de manière spectaculaire mais opposée l'activité des systèmes histaminergiques cérébraux évaluée par la vitesse de renouvellement de l'amine endogène [3, 8]. L'effet activateur attendu du thiopéramide sur ce paramètre révèle que, même dans des conditions physiologiques, les systèmes histaminergiques sont constamment soumis à une rétroaction inhibitrice par le neurotransmetteur qu'ils libèrent. $\mathrm{La}$ levée de ce frein par administration du thiopéramide fournit, pour la première fois, un moyen d'amplifier expérimentalement les transmissions histaminergiques cérébrales.

Une des fonctions les plus probables de l'histamine cérébrale est le maintien d'un état d'éveil. Cette fonction était déjà suggérée par un faisceau d'observations: les neurones histaminergiques sont localisés dans une région de l'hypothalamus dont l'implication dans ces processus était suspectée depuis longtemps, l'injection intracérébrale d'histamine produit un éveil électrographique alors que l'inhibition de sa synthèse ou le blocage des récepteurs $\mathrm{H}_{1}$ (par les antihistaminiques «sédatifs») produit un effet grossièrement inverse. Récemment, ces hypothèses ont reçu une large confirmation par l'emploi, dans le laboratoire du Pr Jouvet, des effecteurs $\mathrm{H}_{3}$ sélectifs chez le chat : la (R) $\alpha$-méthylhistamine augmente la durée du sommeil lent alors que le thiopéramide induit un éveil prolongé chezl 'animal [9]. Chez le rongeur, l'administration d'a-méthylhistamine directement dans le noyau accumbens, que l'on sait être impliqué dans le contrôle de l'activité locomotrice, réduit cette dernière et son effet est levé en présence de thiopéramide [10].

Ces données expérimentales, rapprochées de l'identification récente du récepteur $\mathrm{H}_{3}$ dans le cerveau humain [11], laissent entrevoir que l'agoniste devrait avoir une activité «sédative» chez l'homme. Mais les contours précis de celle-ci (anxiolytique, hypnogène, etc.) sont encore difficilement prévisibles, tant il est vrai que, dans ce domaine, les modèles animaux sont très faiblement prédictifs de l'action thérapeutique de nouvelles classes d'agents. En revanche, les antagonistes $\mathrm{H}_{3}$ devraient exercer une activité stimulante de la vigilance qui serait particulièrement précieuse car, mis à part les amphétaminiques, dont les inconvénients majeurs sont bien connus, la thérapeutique manque singulièrement d'agents exerçant une telle activité. Cette hypothèse est quelque peu étayée par l'observation selon laquelle la $\beta$-histine, médicament utilisé depuis longtemps et avec quelque succès pour améliorer la vigilance de sujets âgés, présente une certaine activité anti-histaminique $\mathrm{H}_{3}$ [12].

Une retombée inattendue de la mise au point des agents $\mathrm{H}_{3}$ sélectifs a été la mise en évidence de la présence de récepteurs $\mathrm{H}_{3}$ sur des systèmes non histaminergiques du cerveau. La cartographie cérébrale des récepteurs $\mathrm{H}_{3}$, entreprise avec une sonde 
radioactive, la ( $\mathrm{R}) \alpha$-méthylhistamine- ${ }^{3} \mathrm{H}$ (figure 1 ), avait déjà révélé que leur distribution ne correspondait pas strictement à celle des projections histaminergiques et cette donnée a été confirmée par des expériences de lésions focalisées (Pollard, Arrang et Schwartz, en préparation). Plus récemment, on a montré que des récepteurs $\mathrm{H}_{3}$ contrôlaient la libération de la sérotonine cérébrale : ces récepteurs ne sont donc pas des autorécepteurs stricts mais jouent aussi un rôle d'hétérorécepteurs présynaptiques [13]. Les implications possibles des systèmes sérotoninergiques dans la régulation des états de vigilance et dans certains syndromes psychiatriques (troubles de l'humeur, psychoses, états agressifs) évoquent des applications élargies des effecteurs $\mathrm{H}_{3}$ en thérapeutique. Ici encore, l'absence de modèles ani- maux prédictifs fiables conduit naturellement à une expérimentation clinique pour vérifier ces hypothèses heuristiques.

\section{Des récepteurs $\mathrm{H}_{3}$ dans l'appareil respiratoire}

Une autre retombée quelque peu inattendue de la découverte du récepteur $\mathrm{H}_{3}$ et de l'utilisation de ses ligands sélectifs a été la mise en évidence de tels récepteurs hors du système nerveux central, où ils avaient été initialement identifiés. En effet, évaluant l'effet de l'administration de (R) $\alpha$-méthylhistamine sur la synthèse d'histamine chez le rat, il a été observé que celle-ci était inhibée non seulement dans le cerveau, mais aussi dans divers tissus périphériques comme le poumon et le tube digestif [3]. Cette réponse met bien en

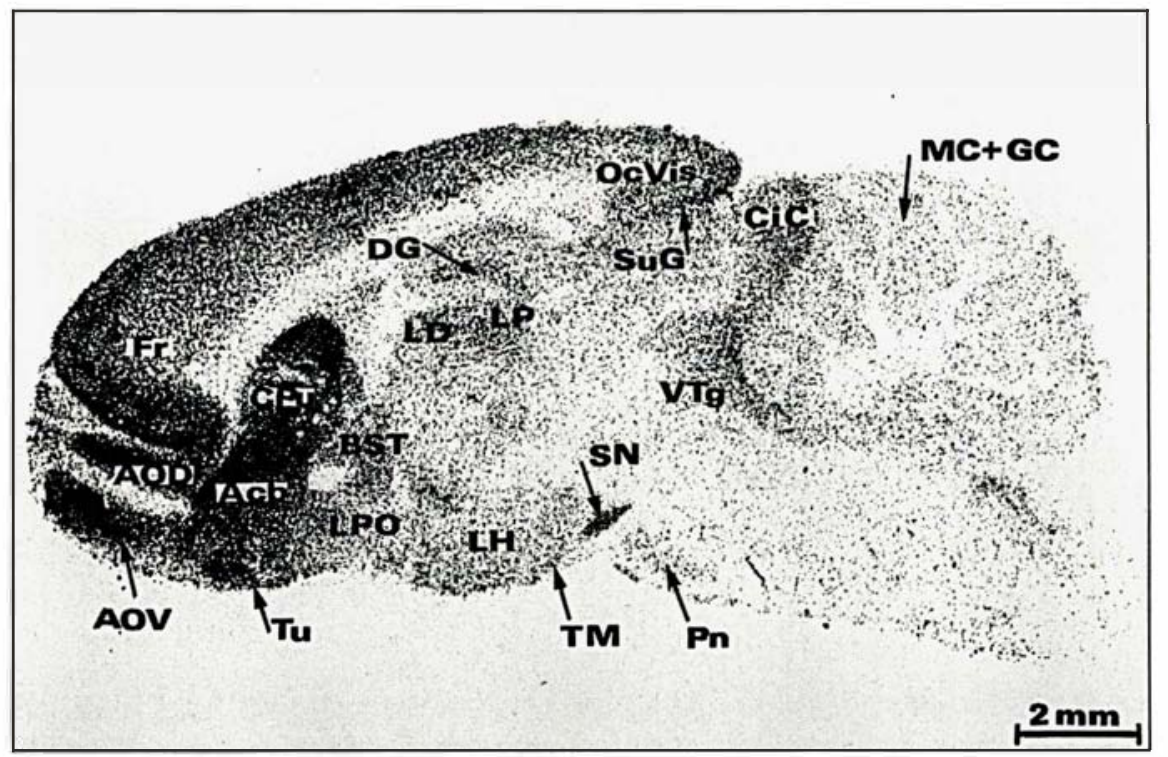

Figure 1. Récepteurs $\boldsymbol{H}_{3}$ : localisation autoradiographique sur une section sagittale de cerveau de rat au moyen de $(R) \alpha$-méthylhistamine- ${ }^{3} H$. $A c b$ : noyau accumbens; $A O D$ : noyau olfactif antérieur, partie dorsale; AOV: noyau olfactif antérieur, partie ventrale; BST: noyau basal de la strie terminale; CPu: noyau caudé putamen; CIC : noyau central du tubercule quadrijumeau inférieur; DG : gyrus denté ; Fr : cortex frontal ; GC : couche granulaire du cervelet ; $L D$ : noyau latérodorsal du thalamus ; $L H$ : aire hypothalamique latérale ; $L P$ : noyau latéral postérieur du thalamus: LPO: aire préoptique latérale; MC: couche moléculaire du cervelet ; Oc Vis : cortex visuel ; Pn : noyau du pont; SN : substance noire; SuG : couche grise superficielle du tubercule quadrijumeau supérieur; TM: noyau tubéromamillaire; Tu: tubercule olfactif; $V T g$ : aire ventrotegmentale latérale. jeu le récepteur $\mathrm{H}_{3}$ car elle est prévenue par le thiopéramide et, en outre, ce récepteur a été clairement identifié comme étant pharmacologiquement identique au récepteur $\mathrm{H}_{3}$ cérébral par des expériences de radioliaison pratiquées sur des membranes pulmonaires de cobaye [3]. L'autoradiographie indique que ces récepteurs sont répartis sur l'ensemble du parenchyme pulmonaire mais que leur densité est plus grande au niveau des bronchioles (figure 2). Cependant la résolution de la méthode autoradiographique utilisée ne permet pas encore d'identifier le(s) type(s) cellulaire(s) qui les expriment.

Il est largement admis que la synthèse d'histamine dans l'appareil respiratoire est l'apanage des mastocytes, cellules clés des processus allergiques et inflammatoires, mais la démonstration définitive que ces cellules expriment le récepteur $\mathrm{H}_{3}$ reste à établir par des approches plus directes. De même, il ne peut être exclu que la synthèse de l'amine et sa modulation par les récepteurs $\mathrm{H}_{3}$ mette en jeu d'autres cellules de l'appareil respiratoire.

Quoi qu'il en soit, des données pharmacologiques récentes ont clairement suggéré la présence et le rôle de récepteurs $\mathrm{H}_{3}$ sur l'innervation de l'appareil respiratoire. On s'aperçoit progressivement que cette innervation et son implication physiopathologique sont beaucoup plus complexes qu'on ne l'imaginait encore il y a quelque années [14]. En bref, l'innervation des voies aériennes par le système nerveux végétatif comporte des voies afférentes ainsi que des voies efférentes et il apparaît que le récepteur $\mathrm{H}_{3}$ est présent sur les unes et les autres (figure 3 ).

C'est la voie efférente vagale excitatrice qui est responsable de la contraction des muscles lisses, de la sécrétion des glandes bronchiques et de la vasodilatation des artérioles pulmonaires. Or, première observation intéressante, à faible concentration, la (R) $\alpha$-méthylhistamine inhibe la contraction de la trachée isolée de cobaye qu'induit la stimulation des fibres cholinergiques pré- et post ganglionnaires [15, 16]. Comme cet effet est reproduit par l'histamine et est compétitivement et sélectivement 
inhibé par le thiopéramide, on est amené à conclure qu'un récepteur $\mathrm{H}_{3}$, pharmacologiquement identique au récepteur cérébral, contrôle l'activité de la voie vagale excitatrice. Fait au moins aussi intéressant, si l'on considère les applications thérapeutiques, l'inhibition des fibres post-ganglionnaires mettant en jeu le récepteur $\mathrm{H}_{3}$ est reproduite sur des bronches humaines isolées [16].

Quant à la voie afférente, son importance fonctionnelle dérive de l'existence de réflexes bronchoconstricteurs locaux ou généraux déclenchés par divers irritants des voies aériennes. L'excitation naît au niveau des terminaisons de neurones afférents primaires localisés dans la paroi des voies aériennes et peut se propager soit par la voie vagale jusqu'aux centres nerveux (noyau du tractus solitaire), soit au moyen d'une fibre récurrente courte (fibre $C$, non myélinisée) jusqu'aux muscles lisses, vaisseaux, glandes bronchiques et épithélium (figure 3). Dans ce dernier cas seraient recrutées, dans un «réflexe d'axone» court, les fibres du système NANC (non adrénergique, non cholinergique) libérant divers neuropeptides (tachykinines telles que la substance $P$, neurokinine A ou le neuropeptide $\mathrm{K}$ ou bien encore le CGRP ou calcitonin gene related peptide). C'est la libération de ces neuropeptides qui entraînerait contraction des muscles lisses, vasodilatation, accroissement de la perméabilité des vaisseaux bronchiques systémiques, sécrétion de mucus et, peut-être, activation de cellules inflammatoires et immunocompétentes [14]. A l'heure actuelle, il est difficile de prévenir ces réactions essentiellement pro-inflamma- toires car, en dépit de recherches actives, des antagonistes puissants de ces divers neuropeptides font toujours défaut. Or l'équipe du Pr P.J. Barnes à Londres a récemment montré que la stimulation des récepteurs $\mathrm{H}_{3}$ réalisée par administration systémique de ( $R) \alpha$-méthylhistamine diminue de manière importante la bronchoconstriction induite chez le cobaye par stimulation du système NANC [17]. Cet effet met bien en jeu un récepteur $\mathrm{H}_{3}$, car il est prévenu par le thiopéramide, mais la localisation précise de ce récepteur reste à établir.

Ces diverses localisations du récepteur $\mathrm{H}_{3}$ dans l'appareil respiratoire paraissent éclairer d'un jour nouveau le rôle de l'histamine dans cet appareil: longtemps considérée comme un médiateur essentiellement proinflammatoire lorsqu'elle agit par

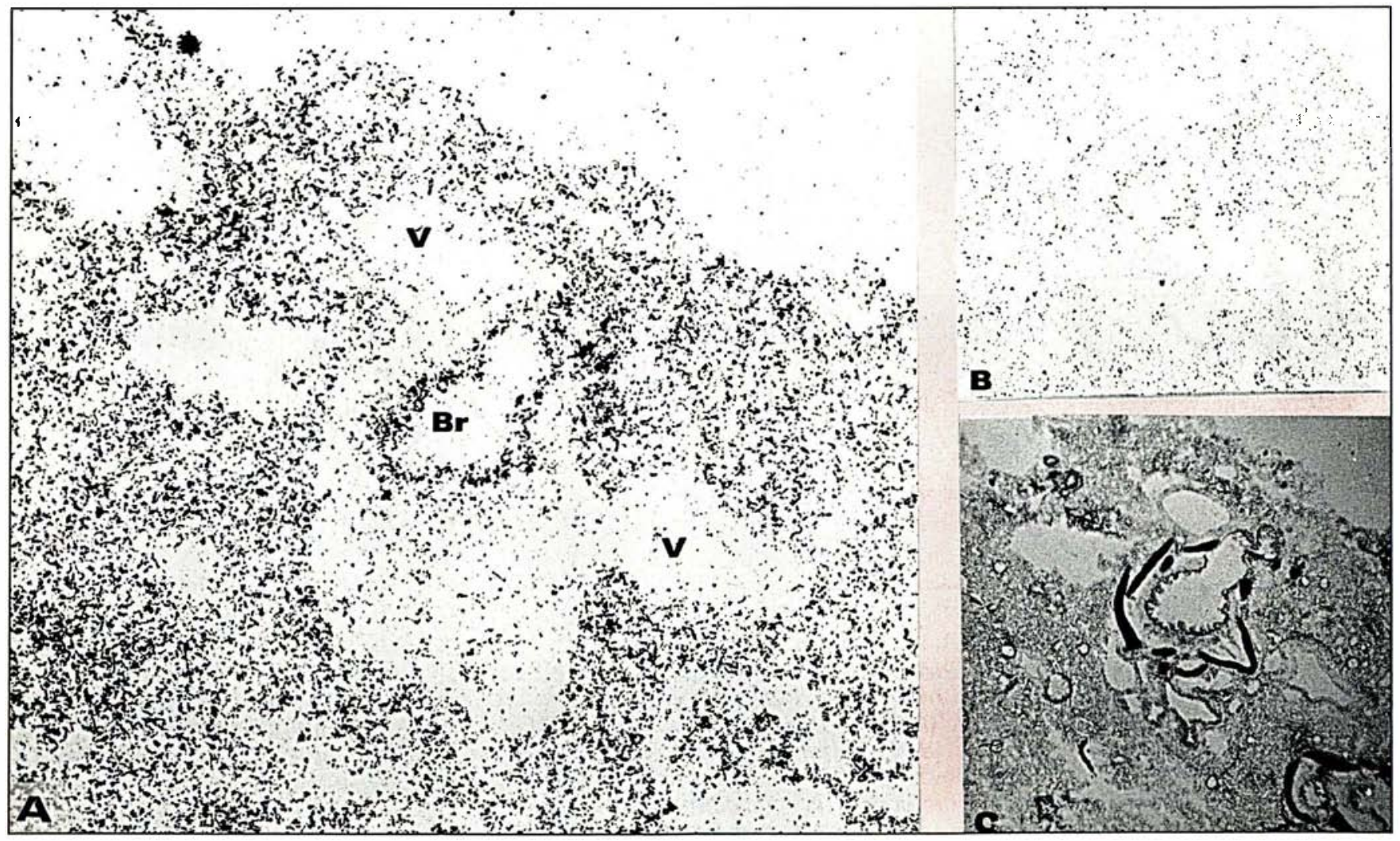

Figure 2. Localisation autoradiographique des récepteurs $\boldsymbol{H}_{3}$ dans le poumon de cobaye. A : liaison totale de la (R) $\alpha$-méthylhistamine tritiée. B: liaison non spécifique (réalisée en présence de thiopéramide). C: histologie. Br: 
l'intermédiaire du récepteur $H_{1}$, il devient maintenant vraisemblable que sa libération locale met simultanément en jeu, par l'intermédiaire du récepteur $\mathrm{H}_{3}$, des régulations de type inhibiteur qui, elles, empruntent des voies neurogènes. L'histamine qui active ce récepteur $\mathrm{H}_{3}$ pourrait trouver son origine dans les mastocytes présents au niveau des ganglions cholinergiques et à proximité des fibres $\mathrm{C}$ sensitives [18].

Il est, dès lors, licite d'imaginer que la stimulation du récepteur $\mathrm{H}_{3}$ pourrait concourir à l'interruption des processus neurogènes et inflammatoires se développant au cours de diverses manifestations pathologiques telles que l'asthme ou la toux.

\section{Des récepteurs $\mathrm{H}_{3}$ dans l'appareil digestif}

Au cours de l'organogenèse, l'ébauche respiratoire est formée par l'évagination de la face antérieure de l'intestin primitif. Dès lors, il n'est pas surprenant de retrouver, au niveau du tube digestif, certaines fonctions attribuées aux récepteurs $\mathrm{H}_{3}$ pulmonaires. Bien que parfois réalisées avec des ligands partiellement sélectifs, diverses études révèlent l'existence du récepteur $\mathrm{H}_{3}$ au niveau des terminaisons parasympathiques et sympathiques.

C'est ainsi que l'enregistrement des potentiels postsynaptiques au niveau de ganglions mésentériques de cobaye a montré que la stimulation des récepteurs $\mathrm{H}_{3}$, ici aussi vraisemblablement présynaptiques, inhibe la libération d'acétylcholine [19]. De même, sur une préparation d'iléon isolé de cobaye, la stimulation du récepteur $\mathrm{H}_{3}$ inhibe la contraction de nature cholinergique induite par stimulation électrique de l'ensemble de la préparation [20]. Ainsi ces deux études montrent, au niveau de l'intestin, la présence de récepteurs $\mathrm{H}_{3}$ présynaptiques jouant un rôle inhibiteur sur la voie efférente parasympathique, analogue à celui qu'ils jouent au niveau pulmonaire. Il est possible qu'une localisation analogue rende compte de l'inhibition par la $(\mathrm{R}) \boldsymbol{\alpha}$-méthylhistamine de la sécrétion acide de l'estomac induite chez le chat par la gastrine [21].

Par ailleurs, une autre étude suggère la présence de récepteurs $\mathrm{H}_{3}$ situés $\mathrm{m} / \mathrm{s} n^{\circ} 6$ vol. 5 , juin 89

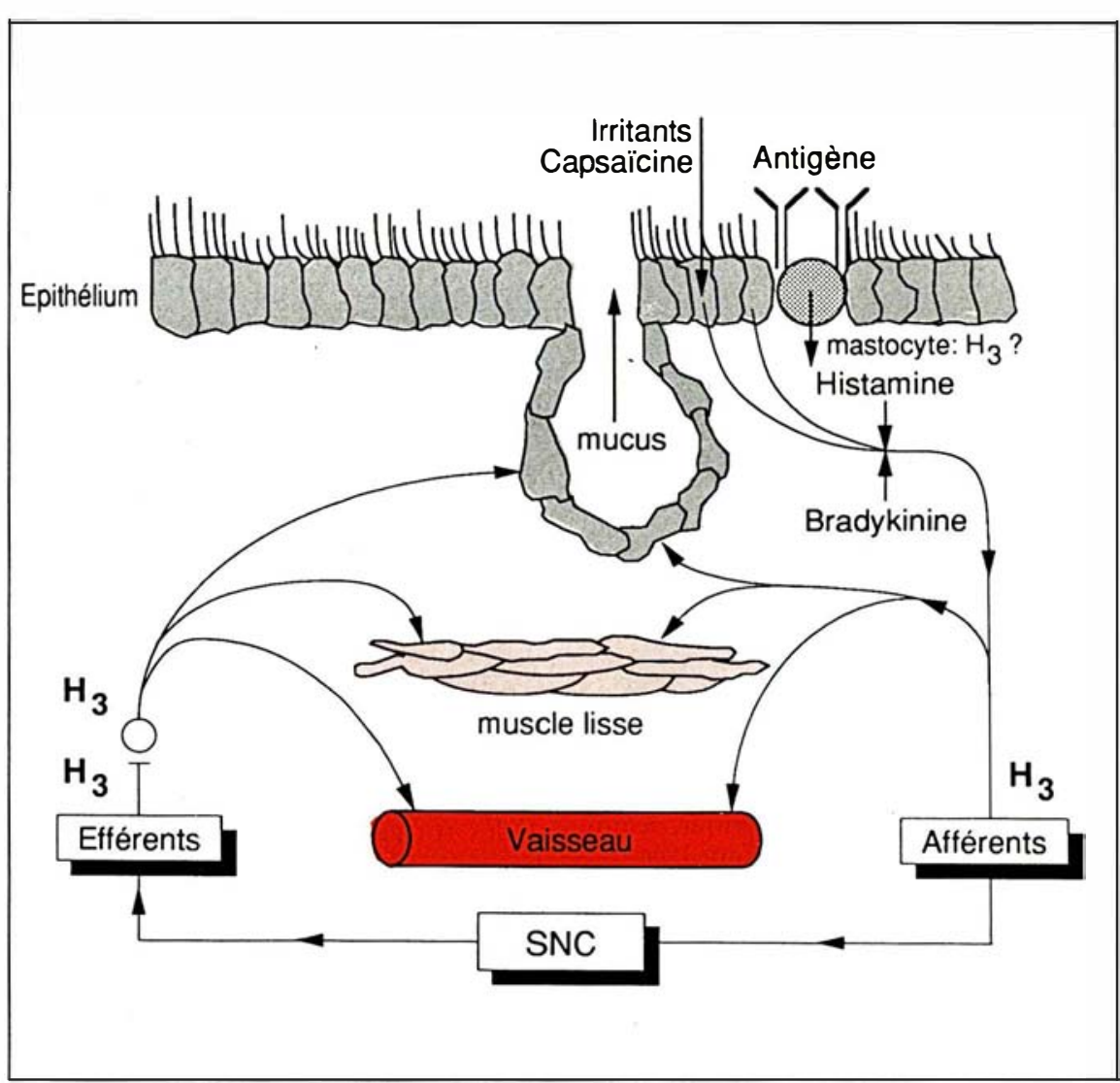

Figure 3. Localisation des récepteurs $\mathrm{H}_{3}$ sur l'innervation des voies aériennes par le système nerveux végétatif. Les irritants inhalés lozone, fumée de cigarette...) ou les médiateurs de l'inflammation libérés lors d'une réaction d'hypersensibilité immédiate, notamment à partir des mastocytes de la muqueuse respiratoire peuvent exciter les terminaisons des neurones afférents primaires. L'excitation de ces terminaisons provoque la libération par un * réflexe d'axone ^ court et un réflexe vagal de neuromédiateurs doués d'activités bronchoconstrictrices et pro-inflammatoires. Des récepteurs $\mathrm{H}_{3}$ situés aux niveaux pré-et post-ganglionnaire, contrôlent l'activité de la voie efférente excitatrice. Des récepteurs $\mathrm{H}_{3}$, dont la localisation est encore mal définie, ont également été mis en évidence au niveau de la voie afférente. La présence de récepteurs $\mathrm{H}_{3}$ sur les mastocytes n'est pas définitivement démontrée.

sur les terminaisons nerveuses sympathiques périvasculaires de l'artère mésentérique de cobaye. La stimulation par l'histamine de ces récepteurs $\mathrm{H}_{3}$ pourrait induire une vasodilatation par inhibition de la neurotransmission sympathique [22]. Cet effet est à rapprocher de la relaxation, endothélium-dépendante, induite par la (R) $\alpha$-méthylhistamine au niveau de l'artère cérébrale moyenne du lapin [23].

Il est encore bien difficile de préciser l'intérêt thérapeutique potentiel en gastro-entérologie de telles propriétés pharmacologiques, mais des effets antispasmodiques et antisécrétoires peuvent être évoqués à la suite de ces études.

\section{Conclusion : du récepteur $\mathrm{H}_{3}$ au médicament}

L'identification du troisième récepteur de l'histamine et des réponses inhibitrices variées qu'induit sa stimulation dans divers organes a déjà mis en lumière une série de fonctions inattendues de cette « vieille » amine. 


\section{RÉFÉRENCES}

14. Devillier P, Matran R, Marsac J. Système non adrénergique, non cholinergique, neuropeptides et asthme. Rev Mal Resp 1988; 5 : $7-20$.

15. Ichinose M, Stretton CD, Schwartz JC, Barnes PJ. Histamine $\mathrm{H}_{3}$-receptors inhibit cholinergic neurotransmission in guinea pig airways. Br J Pharmacol 1989 (sous presse).

16. lchinose M, Stretton CD, Barnes PJ. Histamine $\mathrm{H}_{3}$ receptor inhibit cholinergic neurotransmission in guinea pig and human airways. Proceedings of the American Thoracic Society. Am Rev Respir Dis (sous presse).

17. Ichinose $\mathrm{M}$, Barnes PJ. Histamine $\mathrm{H}_{3}$ receptor mediated inhibition of non-adrenergic non-cholinergic broncho-constriction in guinea pig in vivo. Proceedings of the American Thoracic Society. Am Rev Respir Dis (sous presse).

18. Barnes PJ. Neural control of human airways in health and disease. Am Rev Respir Dis 1986 ; 134 : 1289-314.

19. Tamura K, Palmer JM, Wood JD. Presynaptic inhibition produced by histamine at nicotinic synapses in enteric ganglia. Neuroscience $1988 ; 25$ : 171-9.

20. Trzeciakowski JP. Inhibition of guinea pig ileum contractions mediated by a class of histamine receptor resembling the $\mathrm{H}_{3}$ subtype. J Pharm Exp Ther 1987; 243 : 874-80.

21. Hervatin F, Arrang JM, Bado A, Dubrasquet $M$, Lewin MJM. Involvement of histamine $\mathrm{H}_{3}$-receptors in pentagastrin stimulation of gastric acid secretion in the cat. Proc Chin Acad Sci 1988; 3 : A25.

22. Ishikawa S, Sperelakis N. A novel class $\left[\mathrm{H}_{3}\right]$ of histamine receptors on perivascular nerve terminals. Nature $1987 ; 327$ : 158-60.

23. Ea-Kim L, Oudart N. A highly potent and selective $\mathrm{H}_{3}$ agonist relaxes rabbit middle cerebral artery, in vitro. Eur J Pharmacol $1988 ; 150: 393-6$.
Ces constatations expérimentales suggèrent un certain nombre d'hypothèses physiopathologiques nouvelles et, à ce stade précoce, ouvrent des perspectives thérapeutiques dans des domaines aussi variés que ceux de la neuropsychiatrie, la pneumoallergologie et la gastroentérologie. Parmi les diverses hypothèses mentionnées, lesquelles vont donner lieu à application thérapeutique? Seuls les essais thérapeutiques actuellement en cours vont permettre de répondre avec précision à cette question. Comme à chaque fois qu'un médicament est mis au point à partir d'une cible moléculaire bien déterminée, et non pas d'un modèle animal d'affection humaine, la iâche de l'investigateur clinique est décisive, délicate mais aussi combien exaltante puisqu'elle est susceptible d'aboutir à des traitements entièrement originaux

\section{Summary}

The third histamin receptor: pharmacology and therapeutic applications

The third histamine receptor subtype $\left(\mathrm{H}_{3}\right)$ was initially characterised as an autoreceptor regulating histamine synthesis in and release from cerebral neurons. With the development of highly potent and selective ligands i.e. (R) a-methylhistamine and thioperamide it has been possible to evidence the tonic influence that $\mathrm{H}_{3}$-receptors exert upon the activity of cerebral histaminergic neurons and their role in arousal mechanisms. However, $\mathrm{H}_{3}$-receptors are also present on non-histaminergic neurons in brain as well as in lung or in the gastrointestinal tract. The latter appear to namely regulate in an inhibitory fashion the activity of cholinergic neurons. Hence, it is postulated that clinical trials currently underway might reveal wide therapeutic applications for the novel $\mathrm{H}_{3}$ receptor ligands. 